\title{
Rising evidence for neurological involvement in COVID-19 pandemic
}

\author{
Narghes Calcagno ${ }^{1,2}$ (1) - Eleonora Colombo ${ }^{1,2}$ - Alessio Maranzano ${ }^{1,2} \cdot$ Jacopo Pasquini ${ }^{1,2}$. \\ Ignacio Juan Keller Sarmiento ${ }^{1,2} \cdot$ Francesca Trogu $^{1,2} \cdot$ Vincenzo Silani $^{1}{ }^{\mathbb{B}}$
}

Received: 7 April 2020 / Accepted: 25 April 2020 / Published online: 12 May 2020

(C) Fondazione Società Italiana di Neurologia 2020

Dear Editor,

A novel coronavirus, named severe acute respiratory syndrome coronavirus 2 (SARS-CoV-2), emerged at the end of 2019 and resulted in a rapidly spreading acute respiratory illness epidemic in Wuhan, China. The World Health Organization (WHO) termed this severe respiratory illness Coronavirus Disease 2019 (COVID-19). Since then, COVID-19 has become a pandemic and has affected over 2 million individuals in more than 200 countries and resulted in more than 135,000 deaths worldwide

Clinically, COVID-19 is primarily a respiratory disease marked by fever, cough, and shortness of breath, and in a small percentage of cases, it can lead to severe respiratory complications and death. However, as the number of cases of COVID-19 grows globally and understanding of the disease is unfolding, there are hints that it might have a neurologic component as well.

\section{Coronavirus infections and CNS}

The novel SARS-CoV-2 belongs to the $\beta$-coronavirus cluster [1] and COVID-19 is the third known zoonotic coronavirus disease after SARS and Middle East Respiratory Syndrome (MERS), which are also caused by $\beta$-coronaviruses SARS-CoV and MERS-CoV, respectively. Evidence from former studies of

Narghes Calcagno

narghes.calcagno@unimi.it

Vincenzo Silani

vincenzo.silani@unimi.it

1 Department of Neurology-Stroke Unit and Laboratory of Neuroscience, Istituto Auxologico Italiano IRCCS - Department of Pathophysiology and Transplantation, "Dino Ferrari" Center, Università degli Studi di Milano, Milan, Italy

2 Neurology Residency Program, Università degli Studi di Milano, Milan, Italy
SARS and MERS indicates that human coronaviruses $(\mathrm{CoV})$ do not remain confined to the respiratory system. Instead, they can disseminate damaging several other regions, including the central nervous system (CNS). Indeed, many human coronaviruses are neurotropic and potentially neurovirulent. Possible mechanisms of entry into the CNS include the neuronal retrograde route and hematogenous dissemination. Within the neuronal route, trans-synaptic transfer via peripheral nerve terminals has been well documented for other coronaviruses. An alternative pathway for neuroinvasion implicates connections from mechano- and chemoreceptors in the lung and lower respiratory airways to specific nuclei in the brainstem.

Neurological manifestations were previously observed in patients with SARS and MERS. A group described four patients who developed axonal polyneuropathy, myopathy, or both approximately 3 weeks after the onset of SARS [2], while others have reported large artery ischemic strokes [3]. These findings raise the critical issue of possibly needing to adjust therapeutic strategies in certain patients. Similarly, patients with MERS-CoV infections have a high probability of experiencing neurological complications. A retrospective study of patients with MERS found that symptoms such as generalized fatigue, confusion, and myalgia are relatively common [4]. Another study reported that almost $1 / 5$ of patients show specific neurological complications, including impaired consciousness, paralysis, ischemic stroke, GuillainBarré syndrome, and other neuropathies which appeared 23 weeks after the respiratory symptoms [5].

Given the neurotropic properties of other $\mathrm{CoV}$ and the similarity between SARS-CoV and SARS CoV-2, it is likely that neuroinvasion plays an important role in COVID-19. Notably, earlier this year, a group found that up to one-third of COVID19 patients presented at least one neurological symptom [6]. Most reported symptoms are non-specific like dizziness, headache, fatigue, and myalgia. However, specific symptoms such as acute cerebrovascular disease, seizure, and coma are also described and are more common in severe cases of COVID19. The incidence of neurological features may vary significantly among different study populations. Further studies are 
needed to assess whether the neurological complications are reflective of direct viral injury or due to the secondary or systemic effects of infection. These symptoms may be indicative of intercurrent viral neuroinvasion and based on preliminary evidence, some authors have hypothesized that SARSCoV-2 takes part in the development of respiratory failure [7]. Indeed, experimental studies in transgenic mouse models have provided important insight into the potentially critical role for CNS infection in SARS, suggesting that death may be secondary to neuronal dysfunction in cardiorespiratory centers of the medulla [8].

The pathogenesis of neurological complications in COVID-19 is probably complex and involves a currently unknown set of interactions between viral properties, tissue susceptibility, and host immune response. SARS-CoV-2 has been shown to bind to the angiotensin-converting enzyme 2 (ACE2) receptor which is primarily expressed on epithelial cells of the respiratory and gastrointestinal tracts and is utilized by the virus to enter host cells. However, ACE2 is also detected in the CNS, especially in the brainstem and regions responsible for regulating cardiovascular functions such as the subfornical organ, paraventricular nucleus (PVN), nucleus of the tractus solitarius (NTS), and the rostral ventrolateral medulla. Interestingly, ACE2 is expressed in both neurons and astroglia [9] and the latter may also play a role in facilitating viral spread in the CNS by becoming activated. This hypothesis is corroborated by the rapid dissemination of SARS-CoV1 in the cerebrum while the virus remained undetected in the cerebellum, where the ratio of neural to non-neural cell types is significantly different [10].

Accumulating evidence suggests that a subgroup of patients with severe COVID-19 might develop a "cytokine storm" syndrome with hypersecretion of cytokines, chemokines, and other inflammatory signals which contribute to the progression of critical illness [11, 12]. This state of hyperinflammation may significantly damage the bloodbrain barrier (BBB), which in turn can amplify the neuroinflammatory process. Neuroinflammatory activity associated with functional brain damage may partly explain the reports of clinical cognitive deficits reported in some patients during and after acute pneumonia. Indeed, altered consciousness, cognitive deficits, and behavioral changes may be caused by systemic inflammation associated with prolonged hypoxia and neuroinflammatory damage to specific cortical areas [13].

Taken together, these pieces of evidence prompt curiosity on the viral properties of SARS-CoV-2 and their impact of COVID-19 on the nervous system, but further studies are warranted to shed light on this phenomenon.

Additionally, since the beginning of the epidemic, anosmia and dysgeusia have been observed in patients with COVID-19 and, with the rapid increase in cases, a growing number of patients presenting with anosmia in the absence of other symptoms are being reported. This has been widely shared on medical discussion boards by physicians from all regions managing a high incidence of cases. Iran has reported a sudden increase in cases of isolated anosmia [14], and other colleagues from China, France, and Northern Italy have the same experience [15]. The first record of direct involvement of SARS-CoV-2 in the CNS came from physicians in Beijing Ditan Hospital, China, who reported a case of encephalitis with confirmed presence of the virus in cerebrospinal fluid. More recently, a case of acute necrotizing hemorrhagic encephalopathy was also described [16].

Moreover, neurological comorbidities in COVID-19 are also likely. Acute ischemic stroke often occurs in the elderly, which is one of the most susceptible demographics of severe COVID-19. Coagulation abnormalities, like thrombocytopenia and D-dimer increase, are frequently seen in COVID-19 patients. Thus, the risks and benefits of intravenous thrombolysis and/or arterial embolectomy should be reconsidered in patients with comorbidities of acute ischemic stroke and COVID-19. SARS-CoV-2 is known to bind to the ACE2 receptor on vascular endothelial cells, resulting in abnormally increased blood pressure. Along with platelet and coagulation dysfunctions, the abnormally high blood pressure contributes to the increased risk of intracranial hemorrhage in COVID-19 patients.

Finally, people with neurological conditions such as amyotrophic lateral sclerosis (ALS), Parkinson's disease, and Alzheimer's disease could be at high risk. Patients and their caregivers should be advised to follow specific recommendations during the pandemic. Likewise, patients with autoimmune diseases such as multiple sclerosis and myasthenia gravis are at high risk of severe COVID-19 as well as disease exacerbations.

\section{Conclusion}

As the number of cases of COVID-19 grows globally, neurologists should be vigilant for possible signs of direct and indirect involvement of the nervous system in its entirety. We should also recognize that some patients that recover from COVID-19 may complain of lasting neurological sequelae that may have been overlooked at the time of acute illness. Therefore, long-term follow-up for neurological deficits will be essential in Italy and the rest of the world. A global warning was sent by one of the authors [17].

\section{Compliance with ethical standards}

Conflict of interest The authors declare that they have no conflict of interest.

Ethical approval None. 


\section{References}

1. Chen Y, Liu Q, Guo D (2020) Emerging coronaviruses: genome structure, replication, and pathogenesis. J Med Virol 92:418-423. https://doi.org/10.1002/jmv.25681

2. Tsai LK, Hsieh ST, Chao CC, Chen YC, Lin YH, Chang SC, Chang YC (2004) Neuromuscular disorders in severe acute respiratory syndrome. Arch Neurol 61:1669-1673. https://doi.org/10.1001/ archneur.61.11.1669

3. Umapathi T, Kor AC, Venketasubramanian N, Lim CCT, Pang BC, Yeo TT, Lee CC, Lim PL, Ponnudurai K, Chuah KL, Tan PH, Tai DYH, Ang SPB (2004) Large artery ischaemic stroke in severe acute respiratory syndrome (SARS). J Neurol 251(10):12271231. https://doi.org/10.1007/s00415-004-0519-8

4. Saad M, Omrani AS, Baig K, Bahloul A, Elzein F, Matin MA, Selim MAA, Mutairi MA, Nakhli DA, Aidaroos AYA, Sherbeeni NA, Al-Khashan HI, Memish ZA, Albarrak AM (2014) Clinical aspects and outcomes of 70 patients with Middle East respiratory syndrome coronavirus infection: a single-center experience in Saudi Arabia. Int Soc Infect Dis 29:301-306. https://doi.org/10. 1016/j.jijid.2014.09.003

5. Kim JE, Heo JH, Kim HO, Song SH, Park SS, Park TH, Ahn JY, Kim MK, Choi JP (2017) Neurological complications during treatment of middle east respiratory syndrome. J Clin Neurol (Korea) 13(3):227-233. https://doi.org/10.3988/jen.2017.13.3.227

6. Mao L, Jin H, Wang M, Hu Y, Chen S, He Q, Chang J, Hong C, Zhou Y, Wang D, Miao X, Li Y, Hu B (2020) Neurologic manifestations of hospitalized patients with coronavirus disease 2019 in Wuhan Chia. JAMA Neurology. https://doi.org/10.1001/ jamaneurol.2020.1127

7. Li YC, Bai WZ, Hashikawa T (2020) The neuroinvasive potential of SARS-CoV2 may be at least partially responsible for the respiratory failure of COVID-19 patients. J Med Virol 2(February):1-4. https://doi.org/10.1002/jmv.25728

8. Netland J, Meyerholz DK, Moore S, Cassell M, Perlman S (2008) Severe acute respiratory syndrome coronavirus infection causes neuronal death in the absence of encephalitis in mice transgenic for human ACE2. J Virol 82(15):7264-7275. https://doi.org/10. 1128/jvi.00737-08

9. Gowrisankar YV, Clark MA (2016) Angiotensin II regulation of angiotensin-converting enzymes in spontaneously hypertensive rat primary astrocyte cultures. J Neurochem 138(1):74-85. https://doi. org/10.1111/jnc. 13641

10. Ding Y, He L, Zhang Q, Huang Z, Che X, Hou J, Wang H, Shen H, Qiu L, Li Z, Geng J, Cai J, Han H, Li X, Kang W, Weng D, Liang P,
Jiang S (2004) Organ distribution of severe acute respiratory syndrome (SARS) associated coronavirus (SARS-CoV) in SARS patients: implications for pathogenesis virus transmission pathways. J Pathol 203:622-630. https://doi.org/10.1002/path.1560

11. Huang C, Wang Y, Li X, Ren L, Zhao J, Hu Y, Zhang L, Fan G, Xu J, Gu X, Cheng Z, Yu T, Xia J, Wei Y, Wu W, Xie X, Yin W, Li H, Liu M, Xiao Y, Gao H, Guo L, Xie J, Wang G, Jiang R, Gao Z, Jin Q, Wang J, Cao B (2020) Clinical features of patients infected with 2019 novel coronavirus in Wuhan, China. Lancet 395(10223):497506. https://doi.org/10.1016/S0140-6736(20)30183-5

12. Zhang $\mathrm{C}$ et al (2020) The cytokine release syndrome (CRS) of severe COVID-19 and Interleukin-6 receptor (IL-6R) antagonist Tocilizumab may be the key to reduce the mortality. Int $\mathrm{J}$ Antimicrob Agents:105954. https://doi.org/10.1016/j.ijantimicag. 2020.105954

13. Sasannejad C, Ely EW, Lahiri S (2019) Long-term cognitive impairment after acute respiratory distress syndrome: a review of clinical impact and pathophysiological mechanisms. Crit Care 23:352. https://doi.org/10.1186/s13054-019-2626-z

14. Bagheri SHR et al (2020) Coincidence of COVID-19 epidemic and olfactory dysfunction outbreak. medRxiv. https://doi.org/10.1101/ 2020.03.23.20041889

15. Giacomelli A, Pezzati L, Conti F, Bernacchia D, Siano M, Oreni L, Rusconi S, Gervasoni C, Ridolfo AL, Rizzardini G, Antinori S, Galli M (2020) Self-reported olfactory and taste disorders in SARS-CoV-2 patients: a cross-sectional study. Clin Infect Dis. https://doi.org/10.1093/cid/ciaa330

16. Poyiadji N et al (2020) COVID-19-associated acute hemorrhagic necrotizing encephalopathy: CT and MRI features. Radiol Soc North Am:201187. https://doi.org/10.1148/radiol.2020201187

17. Silani V (2020) Invited commentary: COVID-19 pandemic in Italy: redefining the role of neurology. In: Neurology Blogs Available at: https://blogs.neurology.org/global/invited-commentary-covid-19pandemic-in-italy-redefining-the-role-of-neurology/

The work described has not been published before and it is not under consideration for publication anywhere else. Its publication has been approved by all co-authors.

Publisher's note Springer Nature remains neutral with regard to jurisdictional claims in published maps and institutional affiliations. 